\title{
Pratiques paysannes de la multifonctionnalité Nordeste brésilien et
}

\section{Nouvelle-Calédonie}

\author{
Éric Sabourin, Marcel Djama
}

\begin{abstract}
Peasants practices of multifonctionnality. Two no european cases: northeast of brazil and new Caledonia - This paper considers how multifunctional aspects of agriculture still remains in countries where public or private resources allocation for multifonctionality are not available. After discussing the problems raised by supporting multifonctionality as a national policy in many developing countries, it investigates the way multifonctionality works at the community level. Examples from peasants communities in Brazil and New Caledonia are analysed to illustrate the social, environmental, but also economical integration capacity of non market relationship, generally governed by reciprocity rules. They illustrate, on a local level, the apprehension of agriculture as a public good.
\end{abstract}

\section{Résumé}

L'article concerne l'agriculture des pays où existent des limites à l'allocation de ressources et au recours au marché pour rémunérer d'autres éléments que la production agricole. Le changement d'échelle dans l'analyse de la multifonctionnalité permet d'observer comment les pratiques des agricultures familiales, au Brésil et en Nouvelle-Calédonie, témoignent de la capacité d'intégration, sociale, environnementale, mais aussi économique des prestations non marchandes souvent régies par des règles de réciprocité. Cette approche permet de préciser, au niveau local, l'appréhension de l'agriculture comme un bien public.

\section{Citer ce document / Cite this document :}

Sabourin Éric, Djama Marcel. Pratiques paysannes de la multifonctionnalité Nordeste brésilien et Nouvelle-Calédonie. In: Économie rurale. N²73-274, 2003. La multifonctionnalité de l'activité agricole. pp. 120-133;

doi : https://doi.org/10.3406/ecoru.2003.5394

https://www.persee.fr/doc/ecoru_0013-0559_2003_num_273_1_5394

Fichier pdf généré le 09/05/2018 


\section{$D$ ratiques paysannes de la multifonctionnalité Nordeste brésilien et Nouvelle-Calédonie}

Eric SABOURIN, Marcel DJAMA • CIRAD, département Territoires, environnement et acteurs. Programme agricultures familiales et mondialisation

Cet article propose de discuter des problèmes que soulève dans un certain nombre de «pays en développement », la mise en œuvre d'une politique de soutien à la multifonctionnalité de l'agriculture. En contrepoint des difficultés posées à l'échelon des politiques publiques, nous envisageons les modalités pratiques de traitement des diverses fonctions de l'agriculture par les acteurs ruraux. Ces modalités sont abordées à travers la présentation de situations empiriques issues des zones rurales économiquement marginales du Nordeste brésilien et de la Province Nord de Nouvelle-Calédonie.

La notion de multifonctionnalité de l'agriculture promue par l'Union européenne et un groupe de pays ${ }^{1}$ principalement dans le cadre des négociations conduites à l'Organisation mondiale du commerce (OMC), pose un certain nombre de problèmes.

En premier lieu, elle est associée à une stratégie d'allocation contractuelle de ressources publiques aux agriculteurs afin de rétribuer des fonctions d'intérêt collectif ou public, autres que la production (FAO, 1999 ; Laurent, 1999). La situation des " Pays des Suds " $^{2}$ est certes très hétérogène, mais pour nombre d'entre eux, une telle proposition est difficilement justifiable

1. Le groupe dit des « amis de la multifonctionnalité » regroupe l'Union Européenne, le Japon, la Corée du Sud, la Norvège, l'Ile Maurice, la Suisse. et applicable dans le cadre des politiques de développement rural.

Par ailleurs, les débats suscités par la multifonctionnalité de l'agriculture portent essentiellement sur les objectifs des politiques et sur les instruments de sa prise en compte mais pas sur les catégories qui structurent le débat. Les discussions sur les instruments économiques de reconnaissance de la multifonctionnalité conduisent notamment à isoler les fonctions les unes des autres, à distinguer les productions marchandes des « considérations non marchandes » et à rechercher les modes de valorisation marchande de ces dernières. Ces « lieux communs " $^{3}$ contribuent à l'élaboration d'une vision désincarnée sinon virtuelle de l'activité agricole, éludant les principes sur lesquels elle repose. De fait, toutes les fonctions auxquelles les agriculteurs concourent n'ont pas vocation à être traitées selon la même logique marchande que celle de la production de biens

2. Nous emploierons par commodité la notion floue de « Pays du Sud » pour qualifier les Etats qui relevaient jusque-là du Tiers-Monde, une autre notion floue. Ces catégories englobantes recouvrent, de fait, des trajectoires économiques et politiques très diversifiées. Les études de cas présentées ici n'ont donc pas de valeur paradigmatique.

3. Nous reprenons ce terme dans le sens « aristotélicien » que lui donne Bourdieu et Wacquant (1998), lorsqu'ils évoquent ces « notions ou thèses avec lesquelles on argumente mais sur lesquelles on argumente pas, ou, en d'autres termes, ces présupposés de la discussion qui restent indiscuté... ". 
alimentaires pour le marché, car pour une grande part d'entre elles, ces fonctions relèvent d'un autre type de rationalité socioéconomique. De surcroît, l'opération logique consistant à isoler les fonctions les unes des autres est, pour une large part, artificielle, dans la mesure où, dans nombre de situations agricoles et rurales, ces fonctions sont étroitement articulées de sorte que le fonctionnement même de l'activité agricole repose sur l'hybridation de ressources marchandes et non marchandes.

Opposer les modalités pratiques de traitement des différentes fonctions de l'agriculture par les acteurs ruraux, aux dimensions proprement institutionnelles et économiques de sa reconnaissance par les politiques publiques, peut donner à croire que l'on confronte des registres incommensurables de la multifonctionnalité. Or, la vision strictement institutionnelle de la multifonctionnalité est précisément une conséquence du biais instrumental à travers lequel elle est imposée dans le débat. Il nous semble, à l'inverse, important de convoquer d'autres registres de faits, en rappelant notamment que pour la grande majorité des agriculteurs de la planète, le caractère multifonctionnel de l'agriculture demeure une évidence (Losch et Bonnal, 2000). Que cette multifonctionnalité vécue soit paradoxalement absente des débats qui confrontent « les amis de la multifonctionnalité » à ses détracteurs dans les négociations internationales, constitue une illustration des asymétries qui caractérisent les positions des acteurs dans ces négociations.

Dans cette perspective, la problématique de la multifonctionnalité appréhendée dans ses dimensions empiriques offre l'opportunité de réinvestir un champ de réalité que les approches strictement sectorielles de l'agriculture ne permettent pas de couvrir.

En effet, dans les pays du Sud, il convient de s'interroger sur les conditions de pérennité de l'activité agricole dans un environnement économique et politique extrêmement défavorable. Poser en ces termes, le questionnement sur les rôles et les fonctions de l'activité agricole nous invite à aborder différentes dimensions, ayant trait aux ressources que les ruraux sont à même de mobiliser, et qui excèdent souvent la simple production de biens alimentaires ou les revenus qui en découlent ; à la constitution d'un autre rapport à l'activité agricole que celle privilégiée par les pouvoirs publics ; aux réseaux sociaux et aux institutions dans lesquelles ces activités de production et de circulation des biens se trouvent insérées.

\section{La multifonctionnalité à l'épreuve des politiques publiques}

\section{Les limites des allocations de ressources publiques ou privées spécifiques}

On peut difficilement aborder la problématique de la multifonctionnalité de l'agriculture à partir de localisations et de contextes économiques qui n'ont pas grand chose à voir avec la situation des pays (souvent industrialisés) qui en font la promotion. Pour la majorité de pays du Sud, il est difficile d'invoquer l'octroi de ressources publiques aux fonctions non directement productives quand les aides à la production en faveur des agricultures familiales ou paysannes sont déjà rares, qui plus est dans des régions où l'autosuffisance agricole et alimentaire n'est pas garantie.

Dans nombre de ces pays, les politiques d'ajustement structurel et financier ont conduit au désengagement de l'État de l'appui à l'agriculture, du soutien aux filières agricoles, voire à la privatisation des services (Sabourin, 1999). Quand il subsiste une politique d'aide à l'agriculture familiale, la multifonctionnalité n'est pas reconnue, l'accent étant mis sur l'appui aux fonctions productives.

Les propositions liées à l'allocation de ressources spécifiques aux fonctions non productives de l'agriculture achoppent ainsi sur des impasses diverses (idéologiques, 
logistiques ou stratégiques) quand elles ne reposent pas sur des fondements théoriques et donc politiques, explicites. L'invocation de comportements éthiques universalistes mais souvent déconnectés des réalités ou des valeurs des populations locales ne constitue plus une alternative crédible. Dans beaucoup de pays du Sud, dix ans de discours bien pensants sur le développement durable ont souvent contribué à habiller, voire à déguiser sous de nouveaux atours, les mêmes propositions monétaires libérales dictées par les institutions de financement multilatérales.

Par ailleurs, la mobilisation de ressources privées en substitution des ressources publiques n'est pas chose aisée. L'accès à ces ressources, en particulier collectives ou associatives à travers les fondations, les organisations non gouvernementales et les organisations de producteurs, suppose des formes de coordination là où règne souvent la dispersion. Certes, des initiatives existent à diverses échelles - internationales (Via Campesina, Réseau Agriculture paysanne et mondialisation, Forum social mondial), nationales, régionales mais elles ont un impact relativement limité, et elles ne constituent pas, pour l'heure, un palliatif pérenne à l'absence de financements publics.

Face à ces contraintes, les perspectives que l'on présente souvent comme des alternatives aux aléas de la production agricole renvoient à des stratégies de diversification d'activité et de conquête de nouveaux segments de marchés diversifiés à partir de la valorisation d'attributs territoriaux ou d'avantages spécifiques : tourisme rural, agrobiologie et produits fermiers, production et commercialisation de biomasse, etc. En pratique, ces opportunités restent souvent limitées à des niches ou segments de marchés très spécifiques et sont de ce fait réservées à une minorité de producteurs très concurrentiels. Comme le rappelle Bartra (2000), toutes les formes d'agricultures ne sont pas compétitives ou à même de mobiliser des avantages comparatifs de nature productiviste et commerciale.

Cette réalité à laquelle se heurte la mise en œuvre d'une démarche de valorisation du caractère multifonctionnel de l'agriculture dans nombre de pays du Sud, ne remet pas en cause les principes et les enjeux liés à la rémunération des fonctions non strictement agricoles. A défaut d'en cerner les modalités d'application, elle invite à s'interroger sur les justifications de cette allocation de ressources.

\section{L'agriculture comme un bien public ?}

Pour justifier l'allocation de ressources aux fonctions non productives, Bindraban et al. $(1999)^{4}$ proposent de considérer l'agriculture comme un bien public.

La production d'externalités positives (gestion des ressources naturelles, séquestration de $\mathrm{CO}^{2}$ ) de même que la création ou la prévention d'externalités négatives (coûts d'entretien des biens publics, coûts de la lutte contre les pollutions..) sont autant de fonctions sociales, écologiques, et économiques « globales » qui confèrent à l'agriculture un caractère public qui implique : a) l'accès non exclusif aux biens et services publics - c'est-à-dire la généralisation de la redistribution et la reconnaissance du caractère inaliénable des ressources publiques - ; b) l'indivisibilité des effets induits (positifs ou négatifs) de la production agricole à l'ensemble des populations rurales ou urbaines environnantes.

Bindabran et al. (op. cit.) confirment qu'une généralisation du principe de libreéchange condamnerait de vastes pans de l'agriculture mondiale, sans offrir d'alternative. De fait, la concurrence ne renforce, à terme, la baisse tendancielle des prix

4. Bindraban P., Griffon M., Jansen H. The Multifonctionality of Agriculture : Recognition of Agriculture as a Public Good or Position Against Trade Liberalization. Cirad Ecopol, Nogent-sur-Marne, 1999, 8 p., papier non publié. 
agricoles que si la libéralisation ne concerne que les zones à haut potentiel agricole. Ils proposent donc de ménager la redistribution de la production tout en valorisant les avantages comparés ou spécifiques, non directement productifs, des zones à potentiel agricole limité. En revanche, ces auteurs n'explicitent pas la question de l'interface entre les zones ou les systèmes de production «à protéger » du libre-échange (selon quels mécanismes ?) et celles où celui-ci pourrait se développer (et dans quelles limites ?).

Ce postulat théorique souffre certes du caractère flou de la notion de bien public, notamment parce que cette notion peut se décliner à différents niveaux, du bien public mondial au bien public local -avec tous les problèmes d'articulation afférents (Kaul, 1999). C'est pourquoi il nous semble pertinent de prolonger l'examen des composantes «biens publics et biens communs » de l'activité agricole et paysanne, aux échelles locales et régionales.

La proposition resterait à compléter, en termes de principes et de valeurs capables de dessiner l'interface des différents niveaux. Nous proposons, à cet effet, d'examiner les pratiques économiques de deux formations sociales rurales, issues de contextes latino-américains et océaniens, en considérant la valeur économique et sociale des biens, de leurs transactions, des prestations de services, ainsi que des rapports sociaux qui les fondent. Cette approche nous semble importante afin de replacer au centre de la question de la multifonctionnalité dans les pays du Sud un certain nombre de pratiques qui ne sont pas prises en compte dans le débat tel qu'il est abordé par les pays du Nord : à savoir une politique publique de reconnaissance de fonctions non marchandes ( considérations non commerciales " de la conférence de Doha), non distorsive sur les marchés de produits agricoles, comme le fait par exemple l'OCDE (2001).

\section{Études de cas : Nordeste du Brésil et en pays Kanak (Nouvelle-Calédonie)}

Notre démonstration s'appuie sur deux situations empiriques bien distinctes en termes d'échelles géographiques, de poids démographiques et économiques et de structurations socio-économiques de l'activité agricole, notamment du point de vue de l'articulation aux marchés agricoles nationaux et internationaux. Il ne s'agit pas tant de développer une analyse comparative entre le Nordeste du Brésil et la Nouvelle-Calédonie, mais d'illustrer comment, dans ces deux cas, l'activité agricole constitue un domaine autour duquel viennent s'actualiser d'autres dimensions de la vie sociale, économique ou religieuse. Ce sont autant de fonctions sociales, identitaires, culturelles, environnementales et économiques associées à l'activité agricole et à la gestion d'espaces ruraux, qui présentent souvent un caractère socio-économique ou agro-écologique essentiel aux niveaux local et régional.

\section{Au Nordeste du Brésil, une agriculture paysanne}

Le Nordeste, une des cinq régions administratives du Brésil réunit neuf Etats ${ }^{5}$ pour une superficie de $1542000 \mathrm{~km}^{2}$ et une population de 47 millions sur les 169 millions de Brésiliens (IBGE, 2001). La population de la zone intérieure semi-aride $\left(937000 \mathrm{~km}^{2}\right)$ constitue cependant $33 \%$ de ce total. L'agriculture familiale et paysanne y représente plus de 2,5 millions de familles organisées en communautés rurales, soit environ $40 \%$ des exploitations agricoles de l'ensemble du Brésil (FAO-INCRA, 1996). Dans ces communautés, les relations familiales et inter-familiales, les prestations d'entraide et la gestion des ressources communes sont encore et souvent organisées ou régulées par une logique non marchande de réciprocité (Caron et Sabourin, 2001).

5. Alagoas, Bahia, Ceará, Maranhão, Paraíba, Pernambouc, Piauí, Rio Grande do Norte et Sergipe. 
Nous entendons par réciprocité la dynamique de reproduction du don et de redistribution, génératrice de lien social, identifiée par Mauss (1924) et Polanyi (1944) puis vérifiée dans toutes les sociétés humaines, depuis que Levi-Strauss (1947, 1950) a montré que les structures élémentaires de parenté sont ordonnées au principe de réciprocité (Temple, 1997).

\section{La réciprocité paysanne : \\ une fonction sociale et économique de sécurité alimentaire}

La majeure partie des agriculteurs du Nordeste semi-aride vit aujourd'hui dans des villages ou hameaux appelés sítios ou comunidades. La comunidade, traditionnellement dirigée par un conseil de chefs de famille, régit l'accès aux ressources collectives (terres, pâturages communaux, eau) et aux équipements communs. Elle traite les litiges dans les cas de métayage et règle les pratiques de redistribution et de partage de travail ou de solidarité interfamiliale. Cette solidarité se manifeste à travers des dons d'aliments, d'intrants (semences, etc) et des aides de travail sans retour systématique, en cas de mauvaise récolte, de maladie ou d'accident dans une des familles. Il s'agit-là de gestion de la sécurité alimentaire familiale, locale, voire régionale (via les réseaux de parents et alliés ayant migré en ville) mais également de la sécurité en eau. Chez les paysans du Sertão, on ne refuse jamais l'accès à l'eau, qui tout comme l'hospitalité, peut être étendu aux troupeaux de tiers en cas de sécheresse prolongée.

\section{La gestion des ressources naturelles}

La gestion des ressources naturelles concerne traditionnellement les parcours collectifs sur forêt sèche (caatinga) et les réserves d'eau qui profitent à tous les membres de la communauté. En contrepartie, ceux-ci doivent assumer certains devoirs : participer à l'entretien et au nettoyage, respecter les différents usages des divers points d'eau.
Cette forme de gestion des ressources collectives et des biens communs relève d'une structure de réciprocité plus complexe qu'une entraide ou qu'un simple partage. En effet, la taille des troupeaux variant selon les familles, la ponction qu'ils opèrent sur la ressource commune : eau, pâturages ou travail collectif est différente d'une famille à l'autre. Comment éviter que certains ne s'approprient l'essentiel de la ressource au détriment des autres ? Contrairement aux prophéties de Hardin (1968) proclamant la dégradation inévitable des biens communs par excès d'usage, les paysans du Sertão ont su trouver des modes de gestion des réserves d'eau ou des vaines pâtures, sans en compromettre systématiquement ni l'accès, ni la reproduction. Ils exercent une régulation collective des prélèvements à partir de comportements humains marqués par des normes de réciprocité, de confiance et de prestige (Ostrom, 1998). Cette régulation est d'autant plus efficace qu'elle s'exerce à l'échelle de l'ensemble d'une petite région. Les alliances distantes (mariages exogamiques et compérages) facilitent une répartition des troupeaux sur l'ensemble du territoire régional. La répartition des animaux entre les descendants, dès leur naissance et lors des mariages (via dotation et dot) limite l'accumulation au niveau d'une génération. Il s'agit là de mécanismes devant permettre à chacun (chaque famille qui participe de ce système de réciprocité) de consommer selon ses besoins tout en préservant le renouvellement ou la conservation de la ressource (terroir cultivé, parcours sur forêt sèche, biodiversité végétale, points d'eau, etc.) Ces pratiques de gestion de l'eau et surtout de la forêt sèche ayant permis de maintenir la reproduction de ces ressources, les utilisations productives (pâturages, abreuvement des troupeaux) ne peuvent être dissociées des fonctions sociales (accès à l'eau) et environnementales (conservation de la forêt sèche et des réserves en eau). 


\section{Développer une gestion multifonctionnelle des ressources}

Dans le Sertão brésilien, l'accès gratuit à l'eau des retenues collinaires, aux terres de décrue, aux pâturages communs, à la maind'œuvre de la communauté constitue à la fois un partage et une redistribution des facteurs de production et des ressources. Ces pratiques contribuent à la fois à la production agricole et à d'autres fonctions d'intérêt collectif : sécurité alimentaire, équité sociale et réduction des différenciations socio-économiques, mais aussi préservation et gestion des ressources naturelles. Reconnues et valorisées, ces pratiques de réciprocité et redistribution peuvent $s$ 'articuler en complémentarité avec la fonction productive marchande et être maintenues y compris sous des formes actualisées. Ignorées, combattues, voire même partiellement déstructurées, elles disparaissent sans être substituées par une prise en charge privée (individuelle), marchande ou publique des fonctions d'intérêt collectif, productives ou non productives. De même, les biens collectifs issus de donations qui ne s'inscrivent pas dans la logique des structures de réciprocité deviennent difficiles à gérer. Les redistributions publiques (État, coopération internationale) ou privées (églises, ONG) peuvent engendrer des conflits quant aux droits d'usage et aux devoirs d'entretien, quand elles ne détruisent pas les pratiques de réciprocité en les dévalorisant ou les soumettant à la dépendance de pouvoirs ou d'obligations externes. Il y a alors confusion en matière de responsabilité, sur l'origine et le sens du don et donc sur celui du partage de ces biens communs. Par exemple, depuis l'intervention de l'église et de l'État (19701980), les puits, pompes et barrages dits « communautaires » se sont multipliés dans les communautés rurales. L'organisation de leur entretien était auparavant assurée par le propriétaire de la fazenda ou par le patriarche de la communauté, moyennant une forme spécifique d'entraide. Avec la distribution clientéliste de citernes et de barrages, la rigueur et la motivation pour ces travaux sont souvent moindres ou donnent lieu à discussion. Les conflits et négociations concernant leur entretien sont apparus entre usagers, mais surtout, entre les communautés et les pouvoirs publics. Selon les paysans, l'État devrait assumer le fonctionnement des équipements collectifs qu'il a construit pour un usage public. Ce type d'infrastructure passerait de l'Etat de bien commun à celui de bien public au sens donné par Ostrom et Ostrom $^{6}(1978)$; ce qui met les autorités incapables de garantir leur entretien en difficulté. Le statut de ces infrastructures est ainsi devenu ambigu en matière de droits d'usage et de responsabilités.

Favoriser les conditions juridiques d'une reconnaissance publique des pratiques de réciprocité paysanne et des valeurs qui leur sont associées facilite leur reproduction ou leur modernisation, et la préservation des ressources communes ou publiques concernées. C'est par exemple de cas avec les pâturages communs sur forêt sèche dans la vallée du Rio São Francisco. Suite aux invasions illégales et à l'appropriation privée de leurs vaines pâtures (liées à la spéculation foncière dans les zones d'irrigation), les communautés paysannes ont négocié avec l'État de la Bahia, l'attribution de titres collectifs de propriété de ces communs (Caron et Sabourin, 2001). Cette législation a permis de reconstituer un corps de règles collectives de partage et de responsabilité, via une forme d'organisation nouvelle, l'association communautaire. Il y a bien modernisation, non pas tant des structures, mais du cadre (du réceptacle) de la réciprocité (Sabourin, 2000) qui a permis d'éviter le vol, la privatisation et le déboisement total des aires communautaires de forêt sclérophylle. Il en est de même, au Nordeste, avec les programmes de construction de réserves d'eau multiusages, barrages, citernes, mais

6. Ostrom et Ostrom (1978) définissent les biens publics par leur accès libre à tous et les biens communs comme des biens publics soumis à des restrictions d'accès ou à des droits d'usage. 
surtout retenues collinaires, qui associent des utilisations productives agricoles (abreuvement des troupeaux, irrigation), des usages économiques non-agricoles (pisciculture, pêche, artisanat) et des utilisations sociales (adduction d'eau et usages domestiques et publics) (Caron et Sabourin, 2001).

\section{En pays Kanak : résistances paysannes à la marginalisation économique}

La Nouvelle-Calédonie est une collectivité française du Pacifique jouissant depuis quelques années d'un large statut d'autonomie interne. L'archipel qui s'étend sur une superficie de $18575 \mathrm{~km}^{2}$ est composé d'une Grande Terre (environ $16000 \mathrm{~km}^{2}$ ) et d'îles mineures. Au recensement de 1996, il comptait 197000 habitants, composés pour près de la moitié $(44 \%)$ de mélanésiens autochtones : les Kanaks. Le territoire se caractérise par une faible densité de population (moins de 10 habitants $/ \mathrm{km}^{2}$ ) et par de grandes disparités économiques et spatiales que peine à résorber une politique volontariste de rééquilibrage mise en œuvre par l'Etat français depuis 1988. A la fin des années $1990,60 \%$ de la population est établie dans la capitale - Nouméa - et sa périphérie. Ce pôle urbain concentre l'essentiel des activités économiques et les principales infrastructures (industrielles, services, équipements sociaux et culturels). Ces disparités économiques et spatiales reposent aussi sur une base ethnique. En effet, si la population autochtone kanake représente $44 \%$ de la population, elle ne rassemble que $34 \%$ des actifs. Les Kanaks résident dans leur grande majorité au sein de "tribus " situées en zone rurale. Celles-ci accueillent $84 \%$ des « exploitations » agricoles recensées en Nouvelle-

7. La « tribu » est une entité administrative et territoriale reconnue. Créée par l'administration coloniale à la fin du XIX ${ }^{c}$ siècle, elle est encore présentée comme la reconnaissance administrative de l'organisation mélanésienne. En tant que cadre de résidence et d'appartenance sociale, elle constitue par ailleurs une réalité sociologique significative pour la population kanak.
Calédonie mais l'ensemble de ces exploitations n'occupent que $22 \%$ de la surface agricole utilisée. En dépit d'une faible contribution à la production agricole marchande de la Nouvelle-Calédonie - qui témoigne son articulation marginale au marché - l'activité agricole reste un vecteur fort de l'ancrage rural et de l'enracinement culturel des Kanaks (Djama, 1999).

Dans une économie pourtant fortement administrée et qui accorde d'importants soutiens au secteur agricole (Djama, 1997) l'allocation de ressources publiques aux fonctions non productives de l'agriculture n'est pas à l'ordre du jour. Elle ne fait pas débat au sein d'une profession agricole peu organisée, ni parmi une administration qui reste largement campée sur une vision techniciste et productiviste de l'agriculture. Les principales dispositions de la Loi d'Orientation agricole (LOA) en vigueur en France métropolitaine depuis 1999 ne sont pas appliquées en Nouvelle-Calédonie.

Toutefois, le caractère dual du secteur agricole et les enjeux en terme de cohésion sociale et d'aménagement du territoire qui en découlent, justifie une meilleure prise en compte et une valorisation des modalités particulières à travers lesquelles les ruraux kanaks abordent l'activité agricole dans sa dimension multifonctionnelle. Celle-ci intègre, en particulier, des fonctions économiques dans leur double dimension marchande (commercialisation des produits) et non marchande (sécurité alimentaire, dons et échanges non marchands de produits) ; mais également des fonctions culturelles ou patrimoniales (inscription identitaire des groupes sociaux et des individus) et des fonctions environnementales (gestion des territoires et des ressources naturelles).

\section{Le caractère multifonctionnel de l'agriculture kanake}

Les sociétés kanaks pré-européennes ont été décrites dans la littérature ethnologique comme un archétype de société agraire (Haudricourt, 1964 ; Bensa, 1990). 
Bien qu'ayant subi d'importantes transformations qualitatives (Barrau, 1956 ; Doumenge, 1982), les systèmes horticoles kanaks contemporains conservent leurs principales caractéristiques. L'igname y occupe, aujourd'hui encore, une place tout à fait prééminente d'un point de vue alimentaire et cérémoniel. Plante de prestige, elle est au cœur du système de production et constitue un élément majeur des biens mis en circulation dans le cadre des prestations entre groupes sociaux. Sa plantation, échelonnée entre les mois de juillet et décembre, commande le calendrier agricole. Les moyens techniques mobilisés sont aujourd'hui encore souvent manuels. La tenure foncière est lignagère. Chaque famille étendue dispose de son propre accès foncier et de ses propres outils. Les activités de production agricole s'organisent à l'échelle de la famille étendue (lignage), voir du groupe de résidence. Elles font intervenir plusieurs niveaux de coopération : si le travail dans les jardins s'effectue de manière individualisée (chaque membre adulte de la tribu cultivant seul les parcelles qui lui sont affectées), les travaux lourds (défonçage des billons, préparations des tarodières...) sont effectués dans le cadre de prestations d'entraides qui mobilisent souvent des jeunes du même groupe d'âge. La finalité marchande de la production agricole est loin d'être dominante. Une part de la production est régulièrement commercialisée, souvent sur des marchés de proximité, mais l'essentiel de celle-ci est affecté à la consommation des unités domestiques et à des échanges non monétarisés. Ces échanges prennent deux formes : il s'agit d'abord d'échanges séculiers de produits agricoles (sous la forme de dons - contre dons) ; ou dans le cadre de trocs (produits agricoles contre poissons par exemple). L'autre forme d'échange est cérémonielle : elle intervient à l'occasion des temps forts de la vie sociale (naissances, mariages, deuils) et constitue, au plan des volumes mis en circulation, l'une des principales affectations de produits agricoles.

\section{L'hybridation de ressources marchandes et non marchandes comme condition de reproduction de l'activité agricole?}

La valorisation marchande des produits agricoles ne constitue donc qu'un aspect de la finalité de l'activité de production. Par ailleurs, les producteurs mélanésiens recourent aux marchés locaux pour l'écoulement de ces produits, plutôt qu'aux circuits de commercialisation institutionnalisés qui commandent l'accès au principal marché de consommateurs que constitue le pôle urbain de Nouméa. Cette orientation peut s'expliquer en partie par de multiples contraintes liées à l'éloignement et aux aléas d'un marché dont les modalités d'accès ne sont pas toujours transparentes. Mais cette explication reste partielle : ces marchés locaux ne constituent pas un échelon localisé des circuits territoriaux de distribution. Ils ne sont pas davantage réductibles à une fonction unique de commercialisation. Ils sont aussi (et sans doute avant tout) un espace de sociabilité, d'expression des solidarités locales et donc de réactualisation et de renforcement de la cohésion sociale. Le marché local (en Nouvelle-Calédonie comme ailleurs) est probablement le mode de transaction marchande le moins impersonnel qui soit.

Certains marchés en tribu peuvent apparaître également comme le support de formes de redistribution monétaire. Sur les marchés municipaux, la politique de prix parfois proposée par les équipes municipales - et qui est basée sur les mercuriales de Nouméa - est souvent ignorée et réajustée par les vendeuses (cette activité se décline souvent au féminin) afin de la rendre accessible à une clientèle locale que l'on côtoie en dehors de la scène du marché. Les femmes de la Province Nord expliquent qu'elles pratiquent des prix différents selon le client (occasionnel ou de proximité), selon le lieu et les circonstances (marché en 
tribu, marché municipal, foire provinciale).

C'est la nature de la relation sociale qui détermine la valeur de la transaction et non pas uniquement la valeur marchande du produit. Car une autre caractéristique majeure de ces marchés de proximité est la quasi-indifférenciation entre vendeurs et clients : les rôles sont interchangeables dans la mesure où les vendeurs sont avant tout des producteurs ruraux (Djama et Tyuienon, 2001).

Le fonctionnement des marchés de proximité met en relief le caractère artificiel et incomplet de l'opposition entre les dimensions marchandes et non marchandes de la circulation des biens agricoles. Il faut d'abord rappeler que le modèle abstrait de l'économie de marché et du libre-échange relève plus de la rhétorique que de la réalité, en particulier dans un système économique aussi administré que celui en vigueur en Nouvelle-Calédonie. Dans cette collectivité française d'Outre-Mer comme ailleurs, les subventions agricoles et les aides publiques diverses sont essentiellement captées par une poignée d'agriculteurs, généralement adossés aux pouvoirs locaux et disposant d'un accès privilégié aux réseaux principaux de commercialisation.

Les producteurs agricoles kanaks restent pour la majorité d'entre eux en marge de ce système clientéliste, de sorte que le recours aux marchés de proximité qu'ils animent et investissent pour l'écoulement de leur production, peuvent apparaître comme un palliatif de cette situation d'exclusion.

Toutefois, si les producteurs kanaks vendent régulièrement une partie de leur production sur ces circuits de proximité, cela ne signifie pas pour autant que les logiques qui commandent leur pratique sont régies par les règles du marché.

En effet, cette circulation marchande monétarisée n'alimente pas une dynamique d'accumulation : outre le fait de permettre le maintien d'une activité agricole et d'un ancrage rural en constituant une alternative pour l'écoulement des produits agricoles, elle semble destinée pour une grande part à supporter des activités à caractère social et d'intérêt général. Les marchés de proximité s'intègrent dans des dynamiques de développement local plus larges, dont la clef de voûte repose sur un foisonnant mouvement associatif. Celui-ci assume des services qui ne sont pas pris en charge par la collectivité, ou seulement partiellement, qu'il s'agisse de l'entretien des territoires ruraux mélanésiens (les réserves tribales), de l'entretien des lieux de culte, mais aussi de l'aide aux personnes âgées, de l'encadrement des jeunes, du soutien scolaire, etc. Il offre des modalités d'encadrement social et permet d'atténuer les frustrations que la persistance des inégalités économiques, ethniques, et régionales peuvent engendrer, malgré dix années d'une «politique de rééquilibrage » en NouvelleCalédonie.

Il faut également préciser que les circulations et redistributions monétaires qui alimentent les marchés de proximité proviennent pour une large part des revenus salariaux des membres de la tribu disposant d'un emploi (non agricole pour la plupart), ou des transferts au sein du groupe domestique du salaire des travailleurs kanaks établis en ville. De ce point de vue, les réseaux de solidarité mobilisés constituent une réponse originale aux limites des lois de l'économie de marché ou au modèle paternaliste et inégalitaire en vigueur en Nouvelle-Calédonie. Ces dynamiques signalent aussi un phénomène qui semble bien dépasser le seul contexte kanak : à savoir le fait que, pour nombre d'agriculteurs confrontés au déclin du revenu de l'exploitation et à l'absence de soutien public, la reproduction de l'activité agricole repose, pour une large part, sur la mobilisation de solidarités économiques familiales ou territoriales et l'hybridation de ressources marchandes et non marchandes.

\section{Quels dispositifs de prise en compte de la mutifonctionnalité ?}

Par rapport aux critères de multifonctionnalité habituellement reconnus dans les pays 
du Nord, ces deux exemples, dans des contextes extrêmement différents, montrent l'existence de fonctions non productives ou non agricoles, indissociables des fonctions productives, ce qui élimine l'hypothèse du découplage entre fonction productive agricole et autres fonctions (Shobayashi et Moreddu, 2000).

Par ailleurs, contrairement à la situation des agricultures des pays du Nord, ces prestations ne peuvent être circonscrites à l'échelle de pratiques individuelles ou relevant d'une seule unité de production, puisqu'elles concernent des dispositifs collectifs, inter-familiaux, communautaires ou en réseaux, ce qui limite également le recours à des systèmes de compensation à l'échelle d'exploitations agricoles (Léger, 2000 ; Rémy, 2000).

L'hypothèse de l'évaluation individualisée de ces prestations ou de leur monétarisation, risque de condamner la reproductibilité de ces prestations fondées sur des modes de gestion collectifs (Rémy, op. cit.).

Leur maintien ou leur actualisation dépend donc essentiellement de propositions à une échelle territoriale du type des Opérations groupées d'aménagement foncier (OGAF) comme c'est le cas en NouvelleCalédonie ou d'intervention facilitant la continuité des modes de gestion collectifs à l'exemple de la reconnaissance de la propriété collective des parcours ou des réserves hydriques au Nordeste, ou encore des travaux d'entraide collective pour l'entretien des biens communs.

On se trouve donc confronté à des hypothèses de reconnaissance de fonctions d'intérêt collectif associées à la gestion de biens communs ou de bien publics.

Les mesures de protection ou de différentiation des marchés et des productions agricoles préconisées par Brindraban et al. (1999), pourraient ainsi correspondre à une articulation entre les systèmes de réciprocité et celui de l'échange marchand. Concernant la question de leur interface, une proposition existe déjà pour le marché des biens alimentaires et identitaires (habillement, artisanat, art, habitat). C'est la politique de qualification (de label) qui garantit un procédé, des normes de qualité, l'origine (le nom du producteur) et limite les effets de la concurrence et de la spéculation. Comme le propose Temple (1999), il s'agirait également de préciser la construction d'équivalences de produits et services (entre divers systèmes de réciprocité, comme cela existe déjà sur les marchés de proximité des pays du Sud, mais aussi entre systèmes de réciprocité et systèmes d'échange, marchand ou non).

Ce rapide survol de deux contextes ruraux du Sud, par-delà leurs différences, met bien en évidence le caractère artificiel de la notion d'irréductibilité entre prestations économiques marchandes et non marchandes et la nécessité de catégories explicatives des logiques socio-économiques qui soient plus opérationnelles. Inscrits, quoique de façon périphérique, dans un environnement économique capitaliste, les acteurs ruraux organisent leurs activités et leurs relations autour de deux logiques qui combinent chacune des dimensions marchandes et non marchandes : l'une est gouvernée par le principe de l'échange, l'autre par celui de la réciprocité ou du don. Godbout (2000) et Caillé (2001) ont illustré comment l'économie du don pouvait s'appliquer également aux sociétés modernes occidentales. On retrouve ces deux logiques économiques dans l'ensemble des sociétés humaines, chacune y étant plus ou moins développée selon les buts que l'homme se propose : l'intérêt privé ou les valeurs humaines. On peut donc associer des valeurs spécifiques à chacune de ces deux logiques économiques. On a bien sûr recours à la valeur d'échange, mesurée par l'équivalent monnaie dans le cadre des prestations d'échange et à d'autres valeurs (responsabilité, équité, prestige, amitié, honneur) engendrant diverses formes d'équivalences (matérielles, symboliques) dans le cas de relations dominées par la réciprocité (Temple et Chabal, 1995). 


\section{Réciprocité et multifonctionnalité de l'agriculture}

De même, pour prétendre renforcer la légitimité et les capacités des organisations des producteurs, en particulier pour la gestion de ressources communes, il serait souhaitable que celles-ci puissent choisir entre les deux logiques, entre le modèle de l'échange qu'il soit marchand (la coopérative, l'entreprise) ou non marchand (troc, systèmes d'échanges locaux, économie solidaire, etc.) et celui de la réciprocité. Nous avons vu que la logique de réciprocité peut engendrer des formes d'organisation et de représentation issues de la tradition (conseil des anciens, conseil de clans, communauté villageoise ou lignagère, etc.), mais elle peut aussi s'actualiser via des institutions nouvelles (groupements, associations, etc.) C'est souvent le cas en matière de répartition et de gestion des ressources naturelles ou des biens communs. Il s'agit, par exemple, des relations entre structures foncières et structures de réciprocité, thème brûlant s'il en est, au Brésil comme en Nouvelle-Calédonie, source de conflits par excellence, dans la mesure où il cristallise les réactions identitaires.

Les pratiques et logiques de réciprocité de la majorité des sociétés rurales concernant la gestion des milieux naturels et la production agricole ne dissocient pas la fonction de production des conditions (physiques, agro-écologiques, humaines, techniques, etc.) de cette production, de sa re-production et de la re-distribution (économique, sociale et identitaire) des ressources ou des produits à la collectivité humaine. Affirmer et appuyer le caractère multifonctionnel de l'agriculture et des espaces ruraux peut donc passer par la préservation des conditions politiques, juridiques et techniques permettant l'actualisation de la réciprocité paysanne dans les pays du Sud, mais aussi, la mise en place de nouvelles formes de réciprocité entre ruraux et agriculteurs, entre urbains et ruraux.
Tout en postulant l'agriculture comme bien public, productrice d'externalités positives ou négatives, éventuellement rémunérées/ taxées par le marché, les politiques publiques ou par la communauté internationale, il serait donc pertinent de promouvoir la capacité des agricultures paysannes et familiales à garantir a) la sécurité et la qualité alimentaire ; b) la gestion partagée et la reproduction de ressources naturelles communes ou publiques ; c) la production du lien social à travers des modes d'organisation locale et des marchés de proximité conformes aux structures et aux valeurs de ces sociétés et de leurs territoires spécifiques.

On retrouve bien la nécessité de confronter plusieurs niveaux de compréhension de la notion de multifonctionnalité de l'agriculture : la communauté économique internationale, support de la libéralisation ; la communauté nationale, support de politiques agricoles productivistes ou protectionnistes et le niveau où se nouent les pratiques à la base de la multifonctionnalité : la famille et la communauté locale. Le dialogue social entre ces niveaux a besoin de se construire à partir de réalités et d'espaces qui soient cohérents et de se fonder, si possible, en premier lieu sur des pratiques et des valeurs qui ont du sens à l'échelle des populations concernées.

\section{Conclusion}

Autour de la question de la multifonctionnalité se nouent plusieurs registres d'intelligibilité, qui dépendent pour une large part des échelles d'analyse et des jeux d'intérêt privilégiés : les modes de régulation du commerce international ; l'avenir des politiques publiques nationales de soutien à l'agriculture ; enfin les formes concrètes à travers lesquelles cette multifonctionnalité s'exprime à l'échelle locale, chez les agriculteurs ou au sein des communautés paysannes.

Face aux débats largement ethnocentriques et souvent désincarnés que suscitent les modalités de reconnaissance de la 
multifonctionnalité de l'agriculture par les politiques publiques, il nous semble important de rappeler ici que la multifonctionnalité demeure, pour de larges composantes de la population agricole mondiale, une réalité vécue, ne serait-ce que parce dans bien des situations, la production de biens alimentaires marchands n'est pas la seule finalité de l'activité agricole. Dans nombre de contextes agricoles et ruraux, les dispositifs qui orientent le caractère multifonctionnel de l'agriculture s'inscrivent dans des institutions sociales qui, à l'instar des exemples brésiliens et kanaks, organisent et donnent sens aux rapports sociaux de production et aux mécanismes de circulation des biens agricoles.

Il ne s'agit pas ici de réifier l'image d'Épinal de communautés rurales homogènes et consensuelles. Encore moins d'occulter le développement de phénomènes de pauvreté rurale liés principalement aux déclins des filières agricoles et aux conditions d'insertion des paysans des pays des Sud sur le marché international.

Le concept de multifonctionnalité peut toutefois à certains égards permettre d'élargir la problématique de la « lutte contre la pauvreté » rurale, en relativisant la question du soutien à la seule production agricole.

A partir des situations observées au Nordeste du Brésil et en Nouvelle-Calédonie, il est possible d'isoler trois éléments caractéristiques des modalités qui contribuent à l'expression du caractère multifonctionnel de l'agriculture en l'absence de tout soutien public. Le premier élément concerne l'articulation entre les sphères économiques marchandes et non marchandes. Dans la plupart des manuels d'économie ou d'anthropologie économique, ces deux sphères sont généralement abordées comme étant irréductibles. Or, en pratique, les formations sociales décrites nous signalent la capacité à faire coexister ces deux dimensions du marchand et du non marchand selon un modèle que nous pourrions provisoirement définir sous le vocable d'économie plurielle. Le deuxième élément ren- voie à l'importance des diverses formes de solidarité (familiales, territoriales ou inter générationnelles) dans ces formations sociales, notamment pour pallier les risques économiques ou les défaillances de l'Etat. La notion d'économie solidaire peut être, dans ce contexte, mobilisée à dessein. Enfin un troisième élément renvoie à la question des ressources qui rendent possibles à la fois le caractère multifonctionnel de l'agriculture et les dynamiques de développement local sur lesquelles elles reposent. En l'absence d'allocations publiques ou privées spécifiques, ces ressources proviennent simultanément des revenus tirés de l'activité agricole, de la contribution du bénévolat (notamment pour l'animation de services d'intérêt général), et de revenus provenant d'activité extra-agricole (salariat intermittent ou rapatriement en zones rurales de salaires de travailleurs migrants).

Aussi, avant d'envisager l'allocation de nouvelles ressources publiques rares ou dépendantes de tutelle, il semble important de valoriser les pratiques et ressources existantes : les ressources naturelles communes, les biens communs, les valeurs et connaissances humaines communes et d'éviter le piège de la monétarisation ou de la marchandisation de ces prestations auquel risquent de conduire des définitions trop rapides de la notion de multifonctionnalité et des propositions de politiques publiques associées au découplage des fonctions

Cet article reprend le texte, après expertise et améliorations, de la communication "Approche de la multifonctionnalité de l'agriculture à travers deux exemples non européens : Nordeste brésilien et NouvelleCalédonie ", présentée au colloque SFER "La multifonctionnalité de l'activité agricole et sa reconnaissance par les politiques publiques "(Paris, mars 2002). Les informations originales sont issues des travaux de recherche conduits par les auteurs au Brésil et en Nouvelle-Calédonie. 


\section{RÉFÉRENCES BIBLIOGRAPHIQUES}

Barrau J. L'agriculture vivrière autochtone de la Nouvelle-Calédonie. Commission du Pacifique Sud, Nouméa, 1956.

Bartra A. A donde irán los excluidos si el sistema que los excluye es global y está en todas partes? in « X World Congress of Rural Sociology », Rio de Janeiro, août 2000.

Bensa A. Nouvelle-Calédonie, un paradis dans la tourmente. Gallimard, Paris, 1990 , 192 p.

Bourdieu P., Wacquant L. Sur les ruses de la raison impérialiste. Actes de la Recherche en Sciences Sociales, 1998, 121-122, p. 109-118.

Caillé A. Anthropologie du don. Le tiers paradigme. Desclée de Browers, Paris, 2001, 277 p.

Caron P., Sabourin E. (Eds) Paysans du Sertão. Les mutations des agricultures familiales au Nordeste du Brésil. CIRAD, Montpellier, 2001, coll. Repères, 225 p.

Djama M. New Caledonia : Agrarian Change and Rural Development. in an Unconventional Economy. In VIII Pacific Science Intercongress, Suva, Fidji 11-18 juillet 1997.

Djama M. Transformations agraires et systèmes ruraux mélanésiens en Grande Terre de Nouvelle-Calédonie. Revue d'ethnobiologie, JATBA, 1999, vol. 41 (1), p. 201-224.

Djama M., Mercoiret M.R., Sabourin E. Évaluation du dispositif OGAF (Opération Groupée d'Aménagement Foncier) en Nouvelle Calédonie. IAC-CNASEA, Paris, 2001, 120 p.

Djama M., Tyuienon R. Notes sur les marchés de proximité en Province Nord de Nouvelle-Calédonie. IAC-CIRAD-TERA, Pouembout, Montpellier, 2001, 23 p.

Doumenge J.P. Du terroir à la ville. Les mélanésiens et leurs espaces en NouvelleCalédonie. CEGET-CNRS, Bordeaux, 1982, 490 p.
FAO. Multiples fonctions de l'agriculture et des terres: l'analyse. FAO, Maastricht, 1999.

FAO/INCRA. Perfil da agricultura brasileira. INCRA, Brasília, 1996, 17 p.

Godbout J. Le don, la dette et l'identité, La Découverte/Mauss, Paris, 2000, 190 p.

Hardin G. The Tragedy of the Commons. Science, 1968, (162), p. 1243-1248.

Haudricourt A.G. Nature et culture dans la civilisation de l'igname : l'origine des clones et des clans. In l'Homme, 1964, 4, p. 93-104.

Instituto Brasileiro de Geografia e Estátisticas (IBGE). Censo da população 2000. IBGE, Rio de Janeiro, 2001.

Kaul I. Global Public Goods : a Key to Modern Policy-Making. In Kaul, Grunberg \& Stern (Eds), « Global public goods International cooperation in the 21 th Century », Oxford University Press, New York, 1999.

Laurent C. Activité agricole, Multifonctionnalité, Pluriactivité. Rapport rédigé pour le Ministère de l'Agriculture et de la Pêche dans le cadre du Comité d'Experts sur les Contrats Territoriaux d'Exploitation (CTE). Mimeo, Ministère de l'Agriculture, Paris, 1999, $26 \mathrm{p}$.

Léger F. Les CTE : une approche contractuelle fondée sur la reconnaissance de la localité. Ensa Montpellier, INRA-SAD, 2000, 7 p.

Levi-Strauss C. Introduction à l'ouvre de Marcel Mauss. In Mauss M., « Sociologie et Anthropologie », PUF, Paris, [1950], 1977, $482 \mathrm{p}$.

Levi-Strauss C Les structures élémentaires de la parenté. In «Les cycles de la réciprocité ", chapitre XXVII, Mouton, La Haye, [1947] 1967.

Losch B. La multifonctionnalité : une opportunité pour les pays du sud. In Colloque SFER « La multifonctionnalité de l'activité agricole et sa reconnaissance par les politiques publiques », 22 mars 2002 , Paris, $15 \mathrm{p}$. 
Losch B. ; Bonnal P. (Coord.). La multifonctionnalité de l'agriculture. Rapport du Groupe de Travail Multifonctionnalité, CIRAD, Montpellier, 2000, 12 p.

Mauss M. Essai sur le Don. In « Sociologie et Anthropologie ", PUF, Paris, [1924], 1950.

OCDE. A Synthesis of Country Reports on Jointness Between Commodity and NonCommodity Outputs. In OECD-Agriculture, "Proceedings of the Workshop on multifunctionality", OECD, Paris, 2-3 July 2001.

Ostrom E. A Behavioral Approach to the Rational-Choise Theory of Collective Action. American Political Science Review, 1998, 92, p.1-22.

Ostrom V., Ostrom E. Public Goods and Public Choices. In "Alternatives for Delivering Public Service : Toward Improved Performance", E.S. Savas (Ed.), Westview Press, Boulder, Colorado, 1978, p. 7-49.

Polanyi K. La grande transformation, : aux origines politiques et économiques de notre temps. Gallimard, Paris, [1944],1983, $420 \mathrm{p}$.

Remy J. Contractual Procedures, Democracy \& Environment Co-institution of Land Management Contracts in France. $\mathrm{X}^{\mathrm{c}}$ World Congress of Rural Sociology, Rio de Janeiro, août 2000, 14 p.
Sabourin E. Family Farming Sustainability and Regional Economic Integration in Brazil: Between Debate and Reality. In Dragun A.K. \& Tisdell C. (Dir.) "Sustainable agriculture and Environment: Globalization and Trade Liberalization", Edward Elgar Editor, Cheltenham, Grande-Bretagne, 1999, p. 229-245.

Sabourin E. Peasant Reciprocity Practises in Brazilian Northeast Rural Communities and Recognition of Agriculture Multifunctionality. $\mathrm{X}^{\mathrm{c}}$ World Congress of Rural Sociology, Rio de Janeiro, août 2000, 15 p.

Shobayashi M., Moreddu C. Production, Externality and Public Good Aspects of Multifunctionality. Document OECD (COM/AGR/APM/TD/WP ), Paris, 2000.

Temple D. L'économie humaine. La revue du Mauss, $2^{\mathrm{c}}$ semestre 1997, $\mathrm{n}^{\circ} 10, \mathrm{p} .103-109$. Temple D. Le Marché de réciprocité. Conférence, in [http//dominique.temple. chez.tiscali.fr], 1999.

Temple D., Chabal M. La réciprocité et la naissance des valeurs humaines. L'Harmattan, Paris, 1995, 263 p. 